\title{
FOLFIRINOX for the treatment of colorectal cancer: latest evidence from clinical trials
}

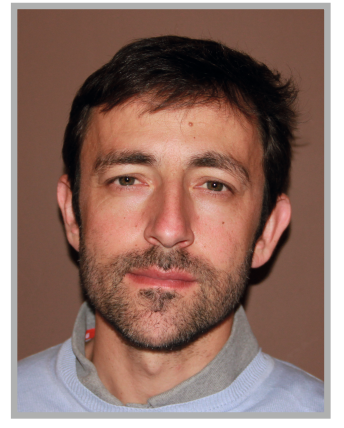

Eric Assenat* “...addition of bevacizumab or cetuximab

to triplet chemotherapy regimens appears feasible and may represent a step

forward in intensive medical treatment of metastatic CRC patients."
Colorectal cancer (CRC) is the second leading cause of cancer-related death worldwide, with more than 200,000 deaths per year in Europe alone [1]. Over half of all patients with CRC will develop metastatic disease, with a quarter having distant metastatic lesions at diagnosis [2].

In the setting of unresectable metastatic CRC the best outcome is achieved in patients receiving fluoropyrimidines oxaliplatin and irinotecan in their course of disease [3]. Over the last decade, several combinations of these three drugs significantly increased response rate (RR) and prolonged overall survival (OS) with a RR of $40-50 \%$ and a median OS of up to 20 months [4]. However, in a sequential strategy, a third of patients are not able to receive a second-line therapy. Several Phase I-II studies demonstrated the feasibility and the promising activity of up-front biweekly fluorouracil infusion combined with oxaliplatin and irinotecan $[5-7]$. A Phase III study comparing FOLFOXIRI to FOLFIRI demonstrated that FOLFOXIRI presented manageable toxicities and significantly improved RR (up to $60 \%$ ), progression-free survival (PFS) and OS [8]. This regimen also increased the rate of secondary resection of liver metastases in patients with initially unresectable lesions $[8,9]$, but did not improve complete response (CR) rate $(<5 \%)$.

In fact, only $10-20 \%$ of patients presenting with colorectal liver metastases (LMs) were considered suitable for liver surgery [10]. However, liver metastasectomy, when feasible, is the only potentially curative treatment, with reported 5 -year and 10-year survival rates of 40 and $25 \%$, respectively [101]. The proportion of patients who can benefit from secondary resection is variable, ranging from 6 to $60 \%$ depending on the type of neoadjuvant combination chemotherapy, the degree of patient selection and reasons for nonresectability [11]. Several authors have emphasized the interest of optimizing firstline chemotherapy schedules to provide a survival advantage. Folprecht et al. demonstrated that tumor response to preoperative chemotherapy is a strong predictor factor for resectability of LM [12]. A recent study evaluating the long-term outcome of 184 CRC patients with LM rendered
"Over half of patients with colorectal cancer will develop metastatic disease, with a quarter having distant metastatic lesions at diagnosis.” 
"Despite potential biases owing to the fact that patients receiving multiple lines of chemotherapy probably experienced a more aggressive disease, overall results indicate that survival can be prolonged when conversion to resectability is achieved after optimal first-line therapy." resectable after neoadjuvant chemotherapy indicated that the patients who were considered cured were those who had responded to firstline chemotherapy $(p=0.05)$ [13]. Likewise, data analysis of the 'LiverMetSurvey' registry from 4408 CRC patients who had undergone liver metastasectomy showed an improvement in survival among the patients operated after one line of chemotherapy compared with those receiving several lines [101]. Despite potential biases owing to the fact that patients receiving multiple lines of chemotherapy probably experienced a more aggressive disease, overall results indicate that survival can be prolonged when conversion to resectability is achieved after optimal first-line therapy.

Thus, it is crucial to deliver from the first-line treatment the most effective therapy for tumor shrinkage and optimal management of CRC patients with LM. In this setting, we have conducted a randomized Phase II trial, METHEP 1 [9], to evaluate whether intensification of firstline chemotherapy (FOLFIRI or FOLFOX vs LV5FU2 with high-dose [HD] irinotecan $260 \mathrm{mg} / \mathrm{m}^{2}$ [FOLFIRI-HD], LV5FU2 with HD oxaliplatin $130 \mathrm{mg} / \mathrm{m}^{2}$ [FOLFOX7] and triplet chemotherapy combining LV5FU2 with irinotecan $180 \mathrm{mg} / \mathrm{m}^{2}$ and oxaliplatin $85 \mathrm{mg} / \mathrm{m}^{2}$ [FOLFIRINOX]) improves secondary resection of initially unresectable colorectal LM. The main concern in most previously published studies is the heterogeneity in definition of unresectability. To avoid such variability, we set out a clear description of unresectability criteria in our protocol, and the reason for unresectability was strictly determined by a panel of surgeons at study entry. In this study, chemotherapy intensification yielded high rates of confirmed tumor response making secondary resection possible in $57 \%$ of patients. Toxicity profiles of intensified regimens were acceptable, and the majority of toxic effects occurred with usual frequency with respect to the drugs used. The incidence of neutropenia was similar in all treatment arms due to primary G-CSF prophylaxis in intensified schedules.

Nevertheless, the concept of chemotherapy intensification has some limitations and should be balanced against the risk of toxicity. Indeed, in the METHEP 1 study, we observed that the occurrence of severe neurotoxicity in the FOLFOX7 arm led to premature chemotherapy discontinuation, and to a worse outcome in this group. The use of chemotherapeutic agents in the neoadjuvant setting is known to increase overall surgical morbidity, and some regimens may have impact on operative mortality. It is also likely that duration of chemotherapy influences the occurrence of postoperative complications. In a recent trial, Karoui et al. demonstrated a postoperative complication rate of $54 \%$ for patients receiving six or more cycles of neoadjuvant chemotherapy versus $19 \%$ for those receiving fewer than six cycles $(\mathrm{p}=0.047)$ [14]. In our study, median time to resection was shorter in the experimental arms compared with the control arms ( 4 vs 6.6 months), and 20 versus $50 \%$ of patients requiring at least eight cycles of chemotherapy prior to surgery, respectively. Overall morbidity rates were found to be slightly higher in the experimental arms, but hepatic failure rates were similar. Interestingly, the FOLFIRINOX arm yielded the best partial $\mathrm{RR}$, the best conversion rate to resectability and a $45 \% \mathrm{R} 0$ resection rate. With a median followup of 50.4 months (range: 1.1-70.9 months), median PFS and OS in this arm were 14.1 months (range: 11.2-21.7 months) and 48.8 months (range: $21.9-$ not reached), respectively. Consequently, we thought that optimal management of CRC patients with formerly unresectable LM should include tailored combination of the most effective chemotherapy regimens and the use of a surgery performed as early as possible to minimize chemotherapy side effects on the liver. In our experience, that was best demonstrated in the FOLFIRINOX arm.

This triplet chemotherapy may provide valuable therapeutic alternative, especially in patients with unresectable CRC LM. Moreover, we have recently confirmed the feasibility of XELIRINOX regimen in a biweekly schedule [15]. Our results are consistent with two recently published data reporting acceptable toxicity profile with similar regimens [16,17]. The recommended dose of $2500 \mathrm{mg} / \mathrm{m}^{2} /$ day on days 1-7 for capecitabine is higher than the dose of $2000 \mathrm{mg} / \mathrm{m}^{2} /$ day proposed by Bajetta et al. [16] and Fornaro et al. [17] from days 2-6 and days $1-7$, when combined with 180 and $165 \mathrm{mg} / \mathrm{m}^{2}$ irinotecan and $85 \mathrm{mg} / \mathrm{m}^{2}$ oxaliplatin, respectively. Interestingly, for other gastrointestinal cancers, Bajetta et al. reported an overall RR of $58 \%$ in a cohort of 12 patients with metastatic gastric cancer treated by biweekly capecitabine with oxaliplatin and irinotecan [18]. Likewise, the Prodige ACCORD 11 trial recently showed that first-line FOLFIRINOX 
tritherapy significantly improved RR, PFS and OS compared with gemcitabine monotherapy among 342 patients with metastatic pancreatic adenocarcinoma [19]. These last results are consistent with the tolerability of FOLFIRINOX regimen even in a fragile population.

Since the METHEP I trial [9] was designed, the interest of adding antiangiogenic therapy to conventional chemotherapy as first-line treatment of metastatic CRC patients has been well established [20-22]. Two Phase III trials showed improvement in both RRs and $\mathrm{R} 0$ resection rates in initially nonresectable patients with KRAS wild-type status given chemotherapy plus cetuximab as compared with those receiving chemotherapy alone [22,23]. Also, in a selected CRC population with unresectable metastases confined to the liver, a $70 \%$ response rate was obtained and R0 resection was achieved in 34\% of the wildtype KRAS tumor patients treated by cetuximab with FOLFOX6 or FOLFIRI [20]. On the other hand, addition of bevacizumab or cetuximab to triplet chemotherapy regimen may represent a step forward in intensive medical treatment of metastatic CRC patients. It adds drug-specific toxicity to that reported with FOLFIRINOX but seems to increase clinical efficacy [24-27].

Given this background, we decided not to proceed to a Phase III trial of FOLFIRINOX versus FOLFIRI/FOLFOX alone. Instead, we initiated a new large randomized Phase II trial to evaluate the efficacy of FOLFIRINOX combined with either cetuximab (in wild-type KRAS) or bevacizumab (in mutated KRAS) in selected CRC patients with initially unresectable metastases confined to the liver. The primary objective will be to compare the secondary resection rate in triplet chemotherapy combination with targeted agents versus doublet chemotherapy (FOLFIRI or FOLFOX) combined with targeted agents according to the KRAS status (PRODIGE 14/ METHEP 2 trial [102]).

\section{References}

1 Boyle P, Ferlay J. Cancer incidence and mortality in Europe, 2004. Ann. Oncol. 16(3), 481-488 (2005).

2 O'Connell JB, Maggard MA, Ko CY. Colon cancer survival rates with the new American Joint Committee on Cancer sixth edition staging. J. Natl Cancer Inst. 96(19), 1420-1425 (2004).

3 Grothey A, Sargent D, Goldberg RM, Schmoll H. Survival of patients with

In summary, the first-line treatment of unresectable metastatic colorectal carcinoma is still a matter of debate. The triplet chemotherapy FOLFIRINOX may provide valuable therapeutic alternative, especially in patients with good performance status and unresectable CRC LMs. Indeed, for these patients, it is crucial to deliver from the first-line the most effective therapy for tumor shrinkage and to perform a LM surgery as early as possible to minimize chemotherapy side effects on the liver. Neoadjuvant intensified chemotherapy demonstrated a superior early tumor response than standard chemotherapy. In this setting, FOLFIRINOX is clearly feasible and particularly interesting in terms of tumor response and secondary resection. This approach does not compromise second-line therapy for these patients, and in the case of unresectable LM persistence after the first-line intensified chemotherapy, the multidisciplinary team should reevaluate the patient case to decide further treatment (conventional chemotherapy plus targeted therapy or intra-arterial hepatic chemotherapy or radioembolization if available). More recently, the addition of bevacizumab or cetuximab to triplet chemotherapy regimens appears feasible and may represent a step forward in intensive medical treatment of metastatic CRC patients. Consequently, we are currently evaluating the FOLFIRINOX regimen in combination with targeted therapies in the randomized Prodige 14/METHEP 2 trial.

\section{Financial \& competing interests disclosure}

The author has no relevant affiliations or financial involvement with any organization or entity with a financial interest in or financial conflict with the subject matter or materials discussed in the manuscript. This includes employment, consultancies, honoraria, stock ownership or options, expert testimony, grants or patents received or pending, or royalties.

No writing assistance was utilized in the production of this manuscript.

advanced colorectal cancer improves with the availability of fluorouracil-leucovorin, irinotecan, and oxaliplatin in the course of treatment. J. Clin. Oncol. 22(7), 1209-1214 (2004).

4 Tournigand C, André T, Achille E et al. FOLFIRI followed by FOLFOX6 or the reverse sequence in advanced colorectal cancer: a randomized GERCOR study. J. Clin. Oncol. 22(2), 229-237 (2004).

5 Ychou M, Conroy T, Seitz JF et al. An open Phase I study assessing the feasibility of the triple combination: oxaliplatin plus irinotecan plus leucovorin/ 5 -fluorouracil every 2 weeks in patients with advanced solid tumors. Ann. Oncol. 14(3), 481-489 (2003).

6 Ychou M, Viret F, Kramar A et al. Tritherapy with fluorouracil/leucovorin, irinotecan and oxaliplatin (FOLFIRINOX): a Phase II study in colorectal cancer patients with nonresectable liver metastases. Cancer Chemother. Pharmacol. 62(2), 195-201 (2008).

7 Masi G, Allegrini G, Cupini S et al. First-line treatment of metastatic colorectal cancer with 
irinotecan, oxaliplatin and 5-fluorouracil/ leucovorin (FOLFOXIRI): results of a Phase II study with a simplified biweekly schedule. Ann. Oncol. 15(12), 1766-1772 (2004).

8 Falcone A, Ricci S, Brunetti I et al. Phase III trial of infusional fluorouracil, leucovorin, oxaliplatin, and irinotecan (FOLFOXIRI) compared with infusional fluorouracil, leucovorin, and irinotecan (FOLFIRI) as first-line treatment for metastatic colorectal cancer: the Gruppo Oncologico Nord Ovest. J. Clin. Oncol. 25(13), 1670-1676 (2007).

9 Rivoire M, Thezenas C, Rebischung F et al. Preliminary results of a randomized Phase II trial comparing standard bi-therapy versus three intensified chemotherapy regimens as treatment for patients with non resectable liver metastases from colorectal cancer (LMCRC) (METHEP). J. Clin. Oncol. 26(Suppl. 20), Abstract 4075 (2008).

10 Benoist $S$, Nordlinger B. The role of preoperative chemotherapy in patients with resectable colorectal liver metastases. Ann. Surg. Oncol. 16, 2385-2390 (2009).

11 Poston G, Adam R, Vauthey JN. Downstaging or downsizing: time for a new staging system in advanced colorectal cancer? J. Clin. Oncol. 24, 2702-2706 (2006).

12 Folprecht G, Grothey A, Alberts S et al. Neoadjuvant treatment of unresectable colorectal liver metastases: correlation between tumour response and resection rates. Ann. Oncol. 16, 1311-1319 (2005).

13 Adam R, Wicherts DA, de Haas RJ et al. Patients with initially unresectable colorectal liver metastases: is there a possibility of cure? J. Clin. Oncol. 27, 1829-1835 (2009).

14 Karoui M, Penna C, Amin-Hashem M et al. Influence of preoperative chemotherapy on the risk of major hepatectomy for colorectal liver metastases. Ann. Surg. 243, 1-7 (2006).
15 Mazard T, Ychou M et al. Feasibility of biweekly combination chemotherapy with capecitabine, irinotecan, and oxaliplatin in patients with metastatic solid tumors: results of a two-step Phase I trial: XELIRI and XELIRINOX. Cancer Chemother. Pharmacol. 69(3), 807-814 (2012).

16 Bajetta E, Celio L, Ferrario E et al. Capecitabin plus oxaliplatin and irinotecan regimen every other week: a Phase I/II study in first line treatment of metastatic colorectal cancer. Ann. Oncol. 18, 1810-1816 (2007).

17 Fornaro L, Masi G, Bursi S et al. A dose finding and pharmacokinetic study of capecitabine in combination with oxaliplatin and irinotecan in metastatic colorectal cancer. Cancer Chemother. Pharmacol. 63, 965-969 (2009).

18 Bajetta E, Verzoni E, Ferrario E et al. Feasibility study of biweekly capecitabine, oxaliplatin, and irinotecan in patients with untreated advanced gastric cancer. Tumori. 95, 43-47 (2009).

19 Conroy T, Desseigne F, Ychou M et al. FOLFIRINOX versus gemcitabine for metastatic pancreatic cancer. $N$. Engl. J. Med. 364, 1817-1825 (2011).

20 Folprecht G, Gruenberger T, Bechstein WO et al. Tumour response and secondary resectability of colorectal liver metastases following neoadjuvant chemotherapy with cetuximab: the CELIM randomised Phase 2 trial. Lancet Oncol. 11, 38-47 (2010).

21 Hurwitz H, Fehrenbacher L, Novotny W et al. Bevacizumab plus irinotecan, fluorouracil, and leucovorin for metastatic colorectal cancer. N. Engl. J. Med. 350, 2335-2342 (2004).

22 Van Cutsem E, Kohne CH, Hitre E et al. Cetuximab and chemotherapy as initial treatment for metastatic colorectal cancer. N. Engl. J. Med. 360, 1408-1417 (2009).
23 Bokemeyer C, Bondarenko I, Makhson A et al. Fluorouracil, leucovorin, and oxaliplatin with and without cetuximab in the first-line treatment of metastatic colorectal cancer. J. Clin. Oncol. 27, 663-671 (2009).

24 Masi G, Loupakis F, Salvatore L et al. Bevacizumab with FOLFOXIRI (irinotecan, oxaliplatin, fluorouracil, and folinate) as first-line treatment for metastatic colorectal cancer: a Phase 2 trial. Lancet Oncol. 11, 845-852 (2010).

25 Assenat E, Desseigne F, Thezenas S et al. Cetuximab plus FOLFIRINOX (ERBIRINOX) as first-line treatment for unresectable metastatic colorectal cancer: a Phase II trial. Oncologist 16, 1557-1564 (2011).

26 Bruera G, SantomaggioA, Cannita K et al. 'Poker' association of weekly alternating 5-fluorouracil, irinotecan, bevacizumab and oxaliplatin (Fir-B/Fox) in first line treatment of metastatic colorectal cancer: a Phase II study. BMC Cancer 10, 567 (2010).

27 Garufi C, Torsello A, Tumolo S et al. Cetuximab plus chronomodulated irinotecan, 5-fluorouracil, leucoverin and oxaliplatin as neoadjuvant chemotherapy in colorectal liver metastases: POCHER trial. Br. J. Cancer 103(10), 1542-1547 (2010).

\section{- Websites}

101 International registry of liver metastases of colorectal cancer. www.livermetsurvey.org/International

102 Chemotherapies associated with targeted therapies on the resection rate of hepatic metastases. http://clinicaltrials.gov/ct2/show/ NCT01442935 